\title{
Numerical simulations for the interaction of the NGC 1333 IRAS 4A outflow and an ambient cloud
}

\author{
Chang Hyun Baek ${ }^{1,2}$, Jongsoo Kim ${ }^{2}$ \\ and Minho Choi ${ }^{2}$ \\ ${ }^{1}$ ARCSEC, Sejong University, Seoul 143-747, KOREA \\ email: chbaek@kasi.re.kr \\ ${ }^{2}$ Korea Astronomy and Space Science Institute, Daejeon, 305-348, KOREA \\ email: jskim@kasi.re.kr, minho@kasi.re.kr
}

NGC 1333 contains numerous young stellar objects and outflows and is a well-studied star formation region. High resolution $\mathrm{SiO}$ observations of the NGC 1333 IRAS 4A region showed a highly collimated outflow with a substantial deflection angle. It was also suggested by the observations that the deflection was due to the interaction of the outflow and a dense cloud core (Choi 2005a, 2005b). In order to make a detailed model of the deflected outflow, we have carried out three-dimensional hydrodynamic simulations of outflow/cloud interactions with a hydrodynamic code based on the TVD scheme. In our models, an initial outflow with number density $10 \mathrm{~cm}^{-3}$ and temperature $10^{4} \mathrm{~K}$ interacts with a spherical cloud with a power-law density distribution. The cloud has a uniform temperature of $10 \mathrm{~K}$ and is surrounded by a homogeneous gas of density $100 \mathrm{~cm}^{-3}$ and temperature $10 \mathrm{~K}$. The radius of the cloud is $0.02 \mathrm{pc}$, and the outflow has a sectional radius $300 \mathrm{AU}$. Through the numerical experiments, we found that the deflection angle is mainly determined by the impact parameter and the density ratio between the outflow and the impact zone of the cloud. The deflection angle is, however, not sensitive to the velocity of outflow. Using initial conditions and parameters which are particularly suitable for NGC 1333 IRAS 4A, we can reproduce its bent morphology. We therefore confirm that the northeastern deflected outflow of the NGC 1333 IRAS 4A may be colliding with a dense cloud core and deflected as a result.

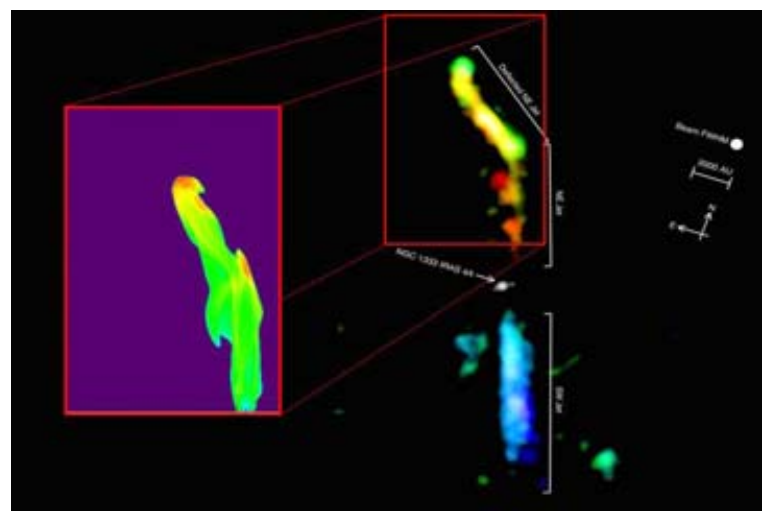

Figure 1. Comparison of deflected outflows seen in observations and simulation. The right box contains an $\mathrm{SiO}$ image toward the northeastern region of the NGC 1333 IRAS 4. A left box shows a map of the column density of shocked gas in our simulation. The deflection angle seen in the simulation is similar to the one seen in the observations. 